\title{
Le robot en chirurgie colorectale : progrès ou mode ?
}

\author{
Y. Panis \\ C Springer-Verlag France 2013
}

Quand la question posée en titre de ce texte concerne un procédé qui n'a jamais démontré un quelconque bénéfice postopératoire dans les quelques études comparatives, pourtant faites par des promoteurs de la technique, et que de toutes façons, il n'existe aucune étude randomisée sur le sujet, on voit bien que la réponse à la question ne pourra être que subjective. C'est donc le parti pris par l'auteur de ce texte et sa réponse est clairement « mode ». Cette mode d'ailleurs a été lancée il y a quelques années par nos amis urologues avec beaucoup de succès et peu d'études.

Mais bien sûr, avant de jeter le bébé avec l'eau du bain, essayons de garder un soupçon d'objectivité...Que dit la littérature dans la rubrique " robot et chirurgie colorectale ", le sujet le plus abordé étant le cancer du rectum, le deuxième étant la rectopexie.

Quand on propose une nouvelle technique chirurgicale, on en espère des avantages (enfin normalement...). Dans l'exérèse totale du mésorectum pour cancer par voie laparoscopique, l'analyse de la littérature récente montre que, " grâce » au robot :

- la durée opératoire est significativement plus longue (les auteurs se rassurant en disant qu'avec l'expérience, on finit par se rapprocher de la durée de la laparoscopie traditionnelle !). Il suffit d'avoir vu dans un congrès international, en direct live, la complexité de l'installation des trocarts pour comprendre que la durée opératoire ne peut être que plus longue...

- les taux de plaie nerveuse, de résection R1, de fistule anastomotique, de morbidité globale, de mortalité, bref tout ce qui pourrait être des bons critères d'évaluation (critères soit dit en passant qui ont évidemment tous été utilisés quand on a comparé la laparotomie à la laparoscopie) sont, à ma connaissance, strictement équivalents par rapport à la laparoscopie traditionnelle dans les

\footnotetext{
Y. Panis $(\bowtie)$

Service de chirurgie colorectale,

Pôle des Maladies de l'Appareil Digestif (PMAD),

Hôpital Beaujon (AP-HP),

100 boulevard du Général Leclerc,

92118 Clichy, France

e-mail : yves.panis@bjn.aphp.fr
}

rares études comparatives (je le répète aucune étude randomisée à ce jour, mais on parle d'une étude qui est en cours..)

- comme il faut un peu tirer dans les coins pour, malgré tout, trouver évidemment un avantage, voici le concept de la « rapidité de la courbe d'apprentissage ». Personne ne rit au fond de la classe. En effet, le robot dans des études récentes réduirait significativement la courbe d'apprentissage. Sans commentaire...

Maintenant, venons en quand même à l'inconvénient majeur, que les utilisateurs ont l'air d'oublier : le coût évidemment. Quelques chiffres : l'achat d'un robot actuel Da Vinci Si 4 bras 1.7 millions d'euros HT ! La maintenance annuelle (sans faire une seule opération) : aux alentours de 150000 euros (première année gratuite, trop sympa...) ! Et je ne parle même pas du matériel pour chaque intervention...

Si on parle de rectopexie, le surcoût matériel est d'environ 500 euros/patient, et si on veut se lancer dans le one trocart (qui serait peut-être finalement la meilleure indication du robot), il faut débourser la modique somme de... 500000 euros! (info Guillaume Meurette, Nantes).

Mais bon, arrêtons de dénigrer cette fantastique machine. Retournons aux méta-analyses afin de voir ce que donnent des études objectives. Cela tombe bien car dans Ann Surg Oncol vient d'être publiée en 2012 une méta-analyse sur 《 robot et chirurgie colorectale » [1] à partir de 16 études comparatives dont 7 spécifiquement sur le cancer du rectum.

En voici les résultats : le robot fait significativement mieux pour les pertes sanguines et pour le taux de conversion en laparotomie. A l'inverse, la durée opératoire et le coût total de l'hospitalisation sont significativement plus longs avec le robot. Enfin, et hélas, car c'est probablement le plus important, tous les critères habituels pour évaluer cette chirurgie (cf. plus haut) sont strictement équivalents entre robot et laparoscopie traditionnelle. Bref, nouvelle déception car aucun bénéfice réellement important.

En conclusion, on ne voit pas trop, avec le robot utilisé aujourd'hui, et à l'heure où les hôpitaux (et le pays tout entier...) traverse une crise sans précédent, pourquoi il faudrait se ruiner pour investir dans l'achat d'une telle machine en chirurgie colorectale. Et si vous avez la chance d'avoir à côté de vous un urologue (qui a donc réussi à convaincre le 
directeur de votre établissement d'en acheter un), demandez lui les études randomisées qui ont clairement validé l'utilisation du robot pour la prostatectomie pour cancer et l'anastomose uréthrovésicale.

Rassurez-vous, ça ne vous prendra pas beaucoup de temps pour les lire : il n'y en a aucune...

\section{Référence}

1. Yang Y, Wang F, Zhang P, et al (2012) Robot-Assisted Versus Conventional Laparoscopic Surgery for Colorectal Disease, Focusing on Rectal Cancer: A Meta-analysis. Ann Surg Oncol Jul 3 [Epub ahead of print] 